Iberian Journal of the History of Economic Thought

ISSN-e 2386-5768

http://dx.doi.org/10.5209//JHE.58090

\title{
Vicent Llombart y el profesor Enrique Fuentes Quintana en la república de los libros
}

Alfonso Sánchez Hormigo ${ }^{1}$

Received: 01/11/2017 / Accepted: 10/11/2017

Resumen. En este artículo, se da cuenta de la importancia de la participación de Vicent Llombart en la colección Economía y Economistas Españoles.

Palabras clave: Vicent Llombart.

[en] Vicent Llombart and the Professor Enrique Fuentes Quintana in the republic of books

Abstract. In this article, the importance of the participation of Vicent Llombart in the collection Economía $y$ Economistas Españoles is stressed.

Keywords: Vicent Llombart.

JEL Classification: B32

Guarda a tu amigo bajo la llave de tu propia vida.

William Shakespeare

El profesor Enrique Fuentes Quintana (Carrión de los Condes, 1924-Madrid, 2007), uno de los más grandes hacendistas españoles de la segunda mitad del siglo XX, dedicó grandes esfuerzos a la investigación y divulgación del ideario de los economistas españoles, fruto de los cuales fue la creación desde el Instituto de Estudios Fiscales, en 1974, de la colección Clásicos del Pensamiento Económico Español. La colección se enmarcaba en un ambicioso proyecto editorial que comenzó a principios de los años setenta impulsado por el doctor Fuentes y que incluía cuatro grandes bloques: las revistas Hacienda Pública Española y Crónica Tributaria; Libros de Bolsillo; Estudios de Hacienda Pública y la citada colección Clásicos del Pensamiento Económico Español.

Igualmente, a finales de los ochenta, diseñó e impulsó la vasta obra en nueve volúmenes Economía y Economistas Españoles en la que contribuyeron cerca de ciento cincuenta investigadores de los que se publicaron más de doscientos trabajos. En ese largo recorrido de más de tres décadas de dedicación a la edición de obras que recogiesen el pensamiento económico de los escritores españoles, estuvo presente el doctor Vicent Llombart —en las dos últimas - como investigador, autor, asesor científico y amigo del profesor Fuentes Quintana.

Como ha quedado reflejado en el Obituario sobre Vicent Llombart incluido en el presente número de IJHET (Cervera 2017), en 1989 recibió la solicitud del profesor Enrique Fuentes Quintana de redactar una síntesis sobre el ideario del conde de Campomanes para ser incorporada a la iniciativa que, más adelante, se transformaría en la colección Economía y Economistas Españoles (Fuentes Quintana (ed.), 1999-2004). El año anterior, había aparecido en la mencionada colección Clásicos del Pensamiento Económico Español del Instituto de estudios Fiscales la edición con un estudio preliminar de Vicent Llombart de la obra de Campomanes Reflexiones sobre el comercio español a Indias (Llombart 1988).

\footnotetext{
Universidad de Zaragoza.

sanchezh@unizar.es

orcid.org/0000-0003-3308-192X
} 
La solicitud del profesor Fuentes volvió a repetirse con motivo del seminario sobre el pensamiento de los economistas asturianos que este último organizó en la Escuela de Estudios Hispánicos de La Granda (Avilés), dirigida por el doctor Juan Velarde, donde estuvieron presentes y fueron oyentes de honor don Severo Ochoa y don Miguel Herrero y Rodríguez de Miñón. Allí, el profesor Llombart abordó el ideario económico del conde de Campomanes. Las respuestas a las solicitudes del doctor Fuentes Quintana se encuentran en el origen de la publicación del profesor Llombart, Campomanes, economista y político de Carlos III, aparecido en 1997.

\section{El proyecto editorial Economía y Economistas Españoles}

En 1984, en el mismo marco de la Escuela de Estudios Hispánicos de La Granda, en un seminario sobre pensamiento económico español e iberoamericano, el profesor Fuentes Quintana esbozó en una pizarra el esquema de un proyecto de investigación que se traduciría en una publicación futura sobre las diversas contribuciones de los economistas españoles desde la época de la Ilustración (partiendo de Jovellanos) hasta nuestros días. Ese fue el germen de Economía y Economistas Españoles. En esa época pidió al profesor Salvador Almenar - en quien depositó su absoluta confianzaque elaborase un documento programático que fundamentase el proyecto desde un punto de vista metodológico. Aunque tal proyecto se retrasó cinco años, el profesor Fuentes Quintana siempre tuvo como soporte e inspiración este importante documento.

En el verano de 1988 pidió a un grupo de investigadores muy cercanos a él que le asesorase en el diseño del proyecto, que finalmente se puso en marcha al año siguiente. Ese grupo lo conformaron los profesores Juan Velarde, José Luis García Delgado, Victorio Valle, Salvador Almenar y Alfonso Sánchez Hormigo, que actuó como coordinador. El proyecto inicialmente se iba a llevar a cabo con la ayuda de la Fundación Fondo para la Investigación Económica y Social de la Confederación de Cajas de ahorro (FIES) y preveía convertirse en dos números de la revista Papeles de Economía Española, de la que era director el profesor Fuentes Quintana. Uno se dedicaría a la recepción de las ideas de las diversas escuelas eco- nómicas y, el otro, al pensamiento económico de los principales autores españoles, ordenados ambos cronológicamente. Más adelante, decidió incorporar investigaciones de historia económica que sirviesen como marco referencial a la recepción de las diversas escuelas, de modo que los cortes tangenciales en la obra no serían dos, sino tres; en su esquema: Historia económica/ Paradigmas/Autores.

El proyecto experimentó diversos momentos de estancamiento, en los que los ánimos permanentes de Hans Meinke, editor de Galaxia Gütenberg y amigo del doctor Fuentes Quintana, y el apoyo incondicional del director de FIES, Victorio Valle, fueron decisivos. A pesar de ello, poco a poco fue aumentando su tamaño, pensándose que desbordaría el marco de la revista, por lo que pasó a convertirse en tres volúmenes que serían editados como libros. Más tarde se convirtieron en cinco $\mathrm{y}$, finalmente, nueve - ocho más uno de cronología, bibliografía e índices- que editarían Galaxia Gütenberg y la Fundación de las Cajas de ahorro (ahora FUNCAS).

Para dar forma definitiva al proyecto, el profesor Fuentes Quintana decidió crear un comité científico en el que estuviesen presentes investigadores relevantes, expertos en el pensamiento económico de los diversos periodos abarcados en la obra. Para ello, llamó a los profesores Ernest Lluch, Manuel Martín Rodríguez, Salvador Almenar, Vicent Llombart, Luis Perdices, Alfonso Sánchez Hormigo y al redactor-jefe de la revista Papeles de Economía Española, Fernando González Olivares. Más adelante, decidió reforzar el comité con algunos buenos conocedores de la historia económica que asesorasen sobre las introducciones de carácter histórico a cada uno de los volúmenes, e integró en el comité a los profesores Pedro Tedde, José María Serrano y Francisco Comín.

De "nuestros hombres en Valencia", Salvador Almenar y Vicent Llombart, el profesor Fuentes consideraba al primero el experto en economía clásica y al segundo en el periodo de la Ilustración; naturalmente, junto a Ernest Lluch y al resto de miembros del comité científico. Nos reuníamos al principio en la sede de la Fundación FIES (más tarde FUNCAS) y en la sala de la Real Academia de Ciencias Morales y Políticas, que presidía, en la que actualmente se conserva el archivo documental que su familia donó a la misma. Fueron largas reuniones, muchas de ellas ocupando las mañanas 
de algunos sábados, en las que se debatió con intensidad sobre la estructura de la obra, los encargos que había que realizar a los diversos investigadores y el contenido de los artículos recibidos.

El peso de Vicent Llombart en las discusiones científicas del comité, a lo largo de la docena de años que duraron las reuniones, fue elevadísimo. Sus estudios, junto a los del profesor Luis Perdices, sobre escritores de economía españoles del periodo de la Ilustración anteriores a Jovellanos, convencieron al director del proyecto de que había que ampliar el espectro temporal de la obra. Era también necesario incluir a los escritores mercantilistas y del periodo de la Ilustración y, previamente, a los autores escolásticos, remontándose incluso al tiempo de la España musulmana.

La confianza del profesor Fuentes Quintana en la solvencia de Vicent Llombart como el mejor especialista en el pensamiento económico de nuestros escritores del siglo XVIII fue absoluta, hasta el punto de depositar en él la responsabilidad de coordinar la edición del volumen tercero de la obra, dedicado al pensamiento de la Ilustración. Fue su editor, además de responsable de la introducción y de cuatro artículos más (dos en solitario y dos en coautoría con los profesores Cervera y Astigarraga).

El profesor Almenar - editor del volumen cuarto-- y él se responsabilizaron de ensamblar las diversas contribuciones, cuyos contenidos se solapaban en casos concretos, tanto en lo relativo a la recepción de las ideas de las diversas escuelas de pensamiento económico como respecto a algunos economistas. Sendas introducciones ejemplifican una labor científica encomiable. La de Vicent Llombart tiene como título: El pensamiento económico de la Ilustración en España (1730-1812) y, en sus algo más de ochenta páginas, presenta un estado de la cuestión como no se había realizado hasta la fecha.

En el mismo volumen incluyó dos importantes colaboraciones: Campomanes, el economista de Carlos III y El "Informe de Ley Agraria" de Jovellanos: núcleo analítico, programa de reformas y fuentes intelectuales. A la decisiva figura de Jovellanos se le dedican en el citado volumen cinco contribuciones que abarcan diversos temas (el Informe de Ley agraria, los viajes de Jovellanos por los caminos de España, sus escritos sobre el comercio exterior o el papel atribuido al Estado, entre otros), pero sin duda las más decisivas fueron la del profesor
Llombart y la del director Fuentes Quintana para quien el economista asturiano era una de las figuras más relevantes entre todos los economistas españoles de la historia - que incluyó un extenso trabajo con el título de "Una aproximación al pensamiento económico de Jovellanos a través de las funciones del Estado". Ambos artículos, junto a las páginas dedicadas a Jovellanos por el profesor Llombart en su introducción al volumen, delinearon su figura y su protagonismo en la España de la Ilustración, así como su influencia en otros escritores posteriores y la trascendencia de su obra.

El profesor Fuentes Quintana anunció al público el proyecto editorial Economía y Economistas Españoles en un seminario de historia del pensamiento económico organizado en homenaje al doctor Fabián Estapé en Zaragoza en 1997, en el que el profesor Llombart presentó una ponencia sobre "Jovellanos proteccionista". Dos años más tarde, en 1999, con motivo de la aparición del primer volumen de la obra, se produjo su presentación oficial. Esta tuvo lugar ese mismo verano en el marco de un seminario organizado en la UIMP por el doctor Fuentes al que invitó a los investigadores que habían sido llamados a colaborar en la obra entre ellos, Marjorie Grice-Hutchinson, Fabián Estapé, Pedro Schwartz y Ernest Lluch - y al que asistieron un buen número de estudiantes.

Allí Vicent Llombart presentó una de las principales ponencias, con el título "El pensamiento económico de la Ilustración: Un panorama sintético", donde efectuó algunos comentarios en relación con los trabajos que había llevado a cabo para el volumen tercero de la obra que ya estaban escritos y verían su publicación junto al volumen cuarto, al año siguiente.

\section{La colección Clásicos del pensamiento económico español}

El impulso de la obra con la aparición de los primeros volúmenes coincidió con la publicación de cuatro títulos en la colección de "Clásicos del Pensamiento Económico Español" que se había frenado a principios de los años ochenta. La colección reapareció en 1989 como resultado de una acción conjunta entre el Instituto de Estudios Fiscales y la Sociedad Estatal Quinto Centenario, que dio lugar a la publicación de diez nuevos volúmenes. Posteriormente, en 1999, el Instituto de Estudios 
Fiscales añadiría otros cuatro títulos a la colección.

Estoy convencido de que fueron ese impulso del proyecto Economía y Economistas Españoles, el interés renovado sobre la figura de Jovellanos y la confianza en el profesor Llombart como investigador sólido de la obra del asturiano, los factores que condujeron al doctor Fuentes Quintana a recuperar con el Instituto de Estudios Fiscales - esta vez desde la Real Academia de Ciencias Morales y Políticas, con la ayuda de la Fundación ICO - la colección que él mismo había creado en 1974.

Soy testigo de que su primer objetivo al impulsar la colección, y así consta en el prólogo al primer volumen editado en esta nueva época (en realidad, la tercera), era publicar una selección de textos de Jovellanos, con un estudio introductorio que naturalmente encargó al profesor Llombart, y otra del gran maestro Antonio Flores de Lemus, aprovechando que su familia había donado su archivo personal en mayo de 1999 a la Real Academia de Ciencias Morales y Políticas. Los tres volúmenes que recogen los escritos seleccionados de Flores de Lemus tuvieron que esperar diez años, por lo que el profesor Fuentes no pudo verlos editados en vida.

Con los Escritos económicos de Jovellanos se alcanzaba el número veinticinco de la colección de clásicos, llevando este último la impronta del autor de su estudio introductorio, el profesor Vicent Llombart, que dedicó la obra al doctor Fuentes Quintana, su director y Presidente de la Real Academia. Basta leer las páginas que redactó el profesor Fuentes Quintana para presentar la colección en esta nueva época, incluidas en el citado volumen, para comprobar el reconocimiento hacia el autor de su estudio introductorio:

Reconocer la oportuna actualidad de la edición de la obra económica de Jovellanos no puede significar, en manera alguna, afirmar su facilidad. Porque esa edición necesitaba un intérprete conocedor profundo de Jovellanos, de su tiempo ilustrado, de su variadísima obra y de la extensa literatura acumulada, a partir de la cual hoy puede y debe conocerse el conjunto de sus aportaciones desde una perspectiva económica rectamente entendida. Para su fortuna, en Clásicos del Pensamiento Económico Español contá- bamos con ese autor capaz de editar la obra económica de Jovellanos cuando se decidió iniciar su nueva época [...]. El ensayo introductorio del profesor Llombart constituye, en verdad, una pieza magistral cuya calidad estoy seguro que sabrán apreciar nuestros lectores.

Con motivo de la reactivación de la colección de clásicos, el profesor Fuentes Quintana convocó a los miembros del comité científico del proyecto Economía y Economistas Españoles, entre los que se contaba el doctor Llombart, para que ellos mismos formasen parte del nuevo consejo asesor de la colección, al que ahora se incorporaron los profesores Juan Velarde y Pedro Schwartz. El director convocó a partir de 2000 a su equipo, que en ocasiones trataba tanto los asuntos relacionados con la aquélla, como los del proyecto Economía y Economistas Españoles, que en estos momentos se encontraba en el ecuador de su publicación. Por ello, el profesor Llombart no se limitó a inaugurar la colección de clásicos con la publicación de los Escritos económicos de Jovellanos, sino que participó muy activamente en la discusión y selección de los textos que se proponía editar, así como de los investigadores a los que se solicitaría la confección de los correspondientes estudios introductorios.

Con posterioridad y, tras un breve paréntesis, aparecieron la Biblioteca de los economistas españoles de los siglos XVI, XVII y XVIII, de Manuel Colmeiro, con un estudio introductorio de los profesores Luis Perdices y John Reeder; una selección de textos krausistas, El krausismo económico español, con un estudio introductorio del profesor José Luis Malo y Escritos monetarios de Raimundo Fernández Villaverde, con un estudio introductorio del profesor José María Serrano. Las obras escogidas de don Antonio Flores de Lemus no verían la luz hasta 2010, tres años después del fallecimiento del doctor Fuentes Quintana, autor del texto introductorio a uno de los volúmenes.

Tras un nuevo lapso de cinco años, con el impulso de los miembros del consejo asesor - del que el profesor Llombart seguía siendo miembro - y el apoyo del actual presidente de la Academia, Juan Velarde y de su académico bibliotecario, Pedro Schwartz, la colección reapareció en el seno de la Real Academia de Ciencias Morales y Políticas, nuevamente en coedición con el Instituto de Estudios Fiscales 
y la Fundación ICO, acometiéndose la edición de nuevas obras: la primera, en 2015, los Discursos y apuntamientos y otros escritos de Mateo Lisón y Viedma, con estudio introductorio de Manuel Martín Rodríguez, y la segunda, los Apuntes de Economía Política y otros escritos de Gabriel Rodríguez, con estudio introductorio de Salvador Almenar, en 2017.

El profesor Llombart, a pesar de ver minada su salud, siguió acudiendo a las reuniones del consejo asesor, en las que manifestó sus opiniones y propuestas - en algún caso retomando las que habían quedado pendientes en la fase anterior de la colección- siempre interesantes y que, en lo relativo a obras de economistas del siglo XVIII, fueron una guía para el resto de los miembros del consejo. Entre sus propuestas figuraron la edición de la obra Discurso sobre Economía Política de Enrique Ramos (seudónimo de Antonio Muñoz), proponiendo que el máximo conocedor de la figura de Enrique Ramos, su discípulo y compañero Pablo Cervera, llevase a cabo la realización de un estudio introductorio, e igualmente que el profesor Pedro Tedde acometiese la publicación de una selección de textos de Francisco Cabarrús.

\section{En recuerdo de don Enrique Fuentes Quintana}

El pasado 6 de junio se cumplieron diez años del fallecimiento del profesor Fuentes Quin- tana. Fueron muchos años de colaboración y aprendizaje para todos los miembros del consejo asesor. Nunca olvidaremos como el profesor Ernest Lluch, cuya afición a comenzar a trabajar muy temprano compartía con el doctor Fuentes, en ocasiones nos decía cuando él no estaba delante: "aprended del profesor Fuentes Quintana". Fue él quien, siendo Presidente de la Real Academia de Ciencias Morales y Políticas en 1999, propuso para académico correspondiente a Vicent Llombart, que siempre sintió un cariño y un respeto especial por él y lloró su muerte, como el resto de los miembros del equipo asesor.

Un año después del deceso, en el verano de 2008, los miembros del comité científico, junto a otros académicos, se dieron cita en la sede del palacio de la Magdalena de la Universidad Internacional Menéndez Pelayo, en un seminario de historia del pensamiento económico organizado por la Fundación Ernest Lluch y el ICO, y dirigido por los profesores Salvador Almenar y Alfonso Sánchez Hormigo. El seminario quería rendir un emotivo homenaje al doctor Fuentes Quintana, revisando la obra Economía y Economistas Españoles. El profesor Llombart le dedicó una cuidada intervención con el título "El pensamiento económico de la Ilustración española: nueva mirada, nuevas aportaciones". Era la mejor manera de homenajearle: recordar su obra y volver creativamente sobre ella, con nuevas aportaciones en un terreno en el que el profesor Llombart fue un maestro.

\section{Bibliografía}

Cervera, P., 2017. "Vicent Llombart Rosa (1948-2017): obituario. Historiografía y compilación bibliográfica", Iberian Journal of the History of Economic Thought, 4 (2), 105-125.

Fuentes Quintana, E. (Ed.), 1999-2004. Economía y economistas españoles. Madrid: Galaxia GütenbergCírculo de Lectores.

Llombart, V. 1988. Reflexiones sobre el comercio español a Indias. 1763. Edición, estudio preliminar y transcripción de Vicent Llombart. Madrid: Instituto de Estudios Fiscales y Ministerio de Hacienda, Clásicos del Pensamiento Económico Español.

- 1992. Campomanes, economista y político de Carlos III. Madrid: Alianza. 